Magnetic ordering and charge dynamics in $\mathrm{k}-(\mathrm{BEDT}-\mathrm{TTF})_{2} \mathrm{Cu}\left[\mathrm{N}(\mathrm{CN})_{2}\right] \mathrm{Cl}$

This content has been downloaded from IOPscience. Please scroll down to see the full text. 2013 J. Phys.: Condens. Matter 25436004

(http://iopscience.iop.org/0953-8984/25/43/436004)

View the table of contents for this issue, or go to the journal homepage for more

Download details:

IP Address: 161.53.9.221

This content was downloaded on 10/10/2013 at $11: 40$

Please note that terms and conditions apply. 


\title{
Magnetic ordering and charge dynamics in $\kappa-(\mathrm{BEDT}-\mathrm{TTF})_{2} \mathrm{Cu}\left[\mathrm{N}(\mathrm{CN})_{2}\right] \mathrm{Cl}$
}

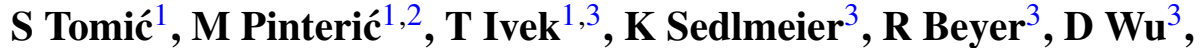 \\ J A Schlueter ${ }^{4}$, D Schweitzer ${ }^{3}$ and $M$ Dressel $^{3}$ \\ ${ }^{1}$ Institut za fiziku, PO Box 304, HR-10001 Zagreb, Croatia \\ ${ }^{2}$ Faculty of Civil Engineering, University of Maribor, Smetanova 17, 2000 Maribor, Slovenia \\ ${ }^{3}$ Physikalisches Institut, Universität Stuttgart, Pfaffenwaldring 57, D-70550 Stuttgart, Germany \\ ${ }^{4}$ Material Science Division, Argonne National Laboratory, Argonne, IL 60439-4831, USA
}

E-mail: stomic@ifs.hr

Received 7 June 2013, in final form 23 August 2013

Published 9 October 2013

Online at stacks.iop.org/JPhysCM/25/436004

\begin{abstract}
The Mott insulator $\kappa$-(BEDT-TTF $)_{2} \mathrm{Cu}\left[\mathrm{N}(\mathrm{CN})_{2}\right] \mathrm{Cl}$ consists of molecular dimers arranged on an anisotropic triangular lattice. At low temperatures a pronounced dielectric anomaly is observed, and eventually a canted antiferromagnetic ground state forms. Optical spectroscopy clearly rules out charge imbalance and the existence of quantum electric dipoles with a dipolar-spin coupling. Here we suggest a novel form of spin-charge coupling where the prominent in-plane dielectric response in $\kappa$-(BEDT-TTF $)_{2} \mathrm{Cu}\left[\mathrm{N}(\mathrm{CN})_{2}\right] \mathrm{Cl}$ is explained by short-range discommensurations of the antiferromagnetic phase in the temperature range $30 \mathrm{~K}<T<50 \mathrm{~K}$, and by relaxation of charged domain walls in the ferromagnetic structure at lower temperatures.
\end{abstract}

(Some figures may appear in colour only in the online journal)

\section{Introduction}

The interplay of electronic correlations and magnetic frustration causes various exotic ground states which have drawn enormous attention in recent years. Some examples are exotic quantum phases on kagome and honeycomb lattices explored in graphene and transition metal compounds [1], but also the Mott insulator and quantum spin-liquid phases found in organic triangular lattices. The realization by organic conductors is especially suitable because they can be nicely tuned by slight chemical and physical variations [2, 3]. The $\kappa$-(BEDT-TTF $)_{2} \mathrm{X}$ materials serve as prime examples in this regard, spanning from the Fermi-liquid metal $\mathrm{X}=$ $\mathrm{Cu}\left[\mathrm{N}(\mathrm{CN})_{2}\right] \mathrm{Br}$, that superconducts below $12 \mathrm{~K}$, to the first realization of a spin-liquid system found in $\mathrm{X}=\mathrm{Cu}_{2}(\mathrm{CN})_{3}$, with no magnetic order down to lowest temperatures despite the strong exchange interaction of $J=250 \mathrm{~K}$ within the triangular lattice. In the present work we take a closer look at the Mott insulator $\mathrm{X}=\mathrm{Cu}\left[\mathrm{N}(\mathrm{CN})_{2}\right] \mathrm{Cl}$, which shows canted-spin antiferromagnetic ordering, i.e., weak ferromagnetism at temperatures below $30 \mathrm{~K}$ [4-7] (although first experiments showed antiferromagnetism below $45 \mathrm{~K}$, all subsequent measurements revealed the antiferromagnetic ordering and canting in the temperature range 20-30 K).

The structure of $\kappa$-phase materials based on the bis-(ethylenedithio)tetrathiafulvalene (BEDT-TTF) molecule consists of two-dimensional layers of dimers arranged in an anisotropic triangular lattice. In particular, $\kappa-(\mathrm{BEDT}-\mathrm{TTF})_{2} \mathrm{Cu}\left[\mathrm{N}(\mathrm{CN})_{2}\right] \mathrm{Cl}$, abbreviated $\kappa-\mathrm{Cl}$, forms an orthorhombic two-layer system (anion layers in $a c$-plane, $b$ axis perpendicular to it) with four BEDT-TTF dimers per unit cell due to the tilting along the $a$-axis in opposite directions for adjacent layers, as displayed in figure 1(a). Conducting layers of cationic BEDT-TTF molecules are separated by essentially insulating anion sheets. The BEDT-TTF donors form face-to-face dimers, which themselves are rotated by about $90^{\circ}$ with respect to neighboring dimers, as sketched in figure 1(b)

With an average of half a hole per molecule, the common theoretical description of $\kappa$-structures considers each dimer as a single site (an effective half-filled band) and applies a Hubbard model with strong on-site Coulomb repulsion $U$. Although it has captured most of the physics [2, 3], this approach was put into 
(a)
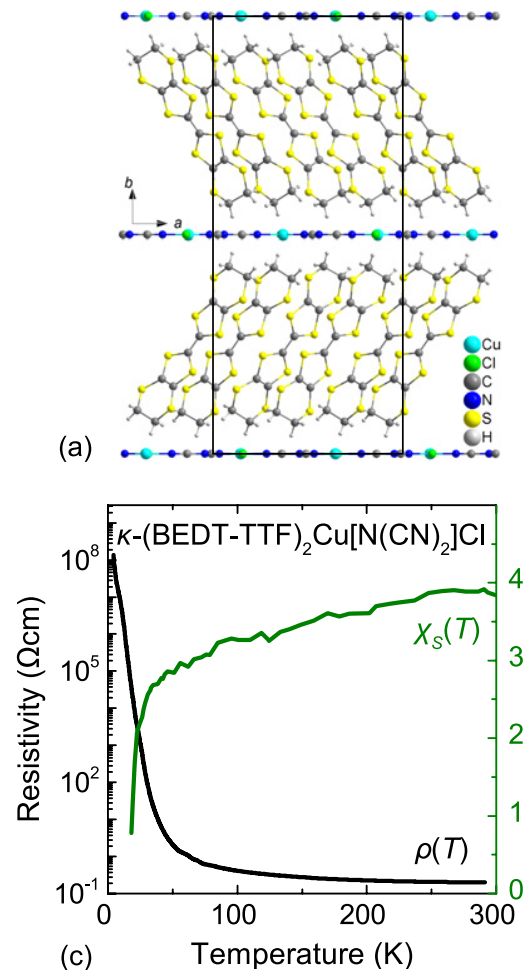

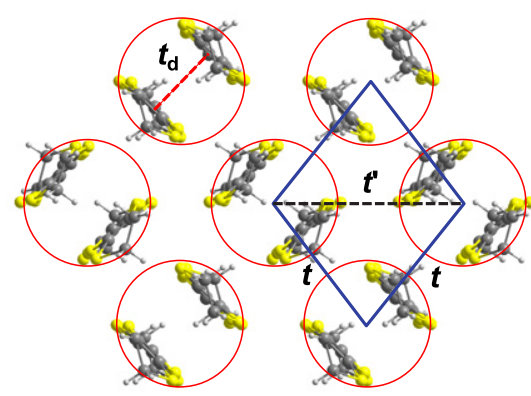

(b)

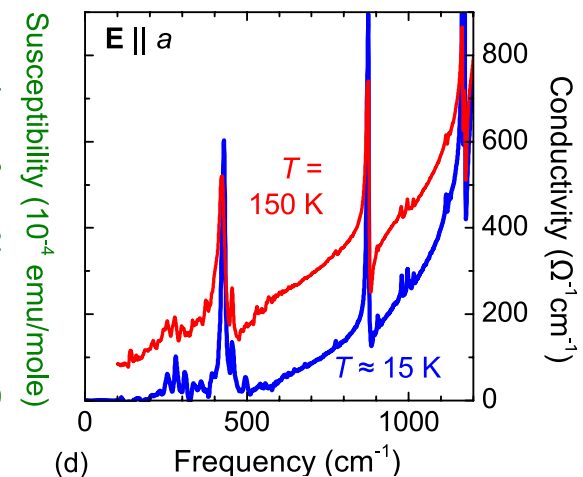

Figure 1. (a) The side view on $\kappa$-(BEDT-TTF $)_{2} \mathrm{Cu}\left[\mathrm{N}(\mathrm{CN})_{2}\right] \mathrm{Cl}$ demonstrates the staggered layers of BEDT-TTF molecules separated by sheets of polymeric anions. (b) For $\kappa$-(BEDT-TTF) ${ }_{2} \mathrm{X}$ in general, the molecules are arranged in dimers which constitute an anisotropic triangular lattice within the plane. The inter-dimer transfer integrals are labeled by $t$ and $t^{\prime}$, and intra-dimer by $t_{\mathrm{d}}$. (c) The temperature dependence of the in-plane dc resistivity $\rho(T)$ evidences an insulating behavior; the magnetic ordering is seen in the susceptibility $\chi_{\mathrm{S}}(T)$ [17]. (d) The optical conductivity shows a Mott gap below $500 \mathrm{~cm}^{-1}$ when cooled down to low temperatures [19].

question recently because dielectric measurements yield certain features in $\kappa$-(BEDT-TTF $)_{2} \mathrm{Cu}_{2}(\mathrm{CN})_{3}[8,9]$ and $\kappa-(\mathrm{BEDT}-\mathrm{TTF})_{2} \mathrm{Cu}\left[\mathrm{N}(\mathrm{CN})_{2}\right] \mathrm{Cl}[10]$ that are interpreted as ferroelectric response caused by charge disproportionation within the dimers. Consequently, several theories were put forward with the aim of explaining these findings. Hotta proposed a dipolar-spin liquid [11] assuming quantum electric dipoles on the dimers that interact with each other through the dipolar-spin coupling resulting in a quarter-filled two-band Hubbard model with inter-dimer Coulomb interaction $V$. She showed that dipolar fluctuations measured by intra-dimer transfer integral $t_{\mathrm{d}}$ are suppressed by $V$, which leads to significant decrease of magnetic correlations. A similar conclusion was reached by Naka et al, who demonstrated that magnetic and ferroelectric phases exclude each other [12]. Notably, Tocchio et al [13] argued that, contrary to previous statements, the magnetic phase can be stabilized in the presence of charge fluctuations. Considerations based on the quarter-filled extended Hubbard model found that only for non-zero electron-lattice coupling the ferroelectricity due to unequal charge distribution within dimers is stabilized in a spin-singlet state $[14,15]$. Finally, a recent $a b$ initio study of $\kappa$-phase materials applying a single-band extended Hubbard model [16] revealed that charge fluctuations (in the metallic state) may also be enhanced by the inter-dimer Coulomb interaction. Note, however, that effects of charge fluctuations within dimers were not taken into account in the latter work.
All these studies indicate the important role of spin-charge coupling in the formation of the low-temperature phase in the charge and spin sectors; however, it has not become clear yet whether any of these theoretical models is appropriate to describe the intriguing physics of the dimerized $\kappa-\mathrm{Cl}$. For this reason, it is of particular interest to learn from experiments more about the origin of the dielectric anomaly in the presence of magnetic order. Our dielectric and optical experiments exclude the presence of electric dipoles within BEDT-TTF dimers. Rather, they support an alternative scenario in which charged domain walls formed in a magnetically ordered background respond collectively to applied external ac electric field. We suggest that the second-order spin-dipolar coupling may play a crucial role and cause a suppression of the dipolar order once the spin order is established at long-range scales below $25 \mathrm{~K}$.

\section{Experimental results}

The spin and charge response of $\kappa-\mathrm{Cl}$ is demonstrated in figures $1(\mathrm{c})$ and (d): upon cooling a crossover takes place from a semiconducting phase to a Mott insulator at $T_{\mathrm{M}} \approx$ $40 \mathrm{~K}$; the antiferromagnetic ordering can best be seen as a change in the spin susceptibility [17]. The in-plane optical conductivity vanishes for low frequencies and temperatures. When extrapolating $\sigma(\omega)$ linearly to zero, we may identify the Mott gap around $500 \mathrm{~cm}^{-1}$ at $T=15 \mathrm{~K}[18,19]$. 


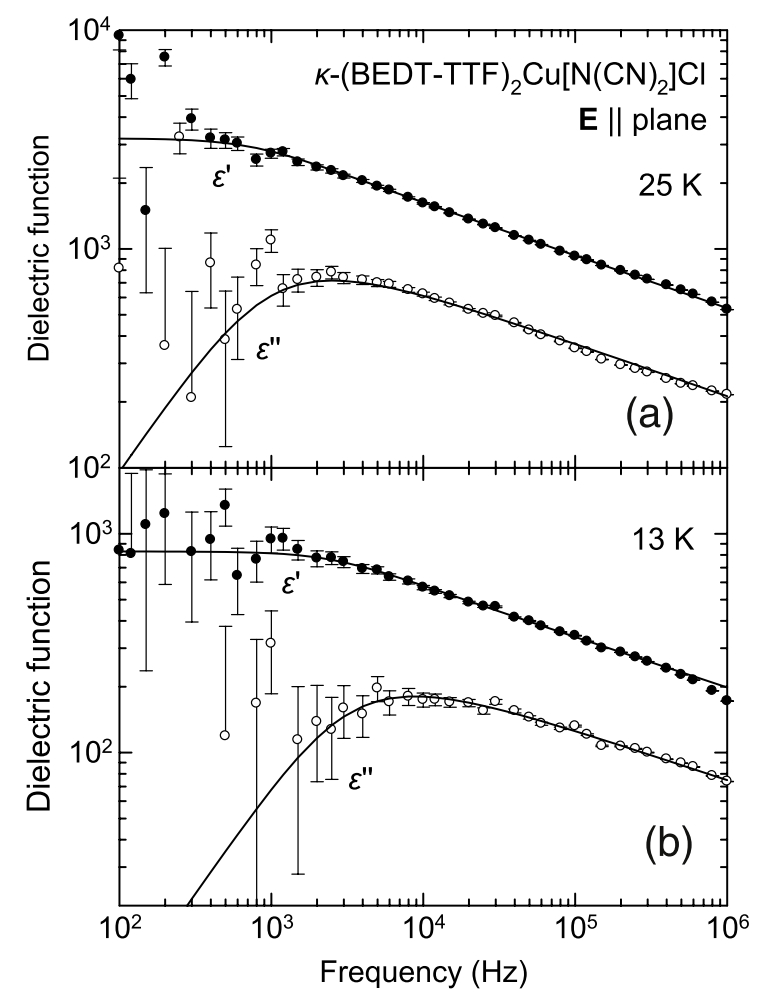

Figure 2. Real and imaginary parts of the in-plane dielectric function of $\kappa$-(BEDT-TTF $)_{2} \mathrm{Cu}\left[\mathrm{N}(\mathrm{CN})_{2}\right] \mathrm{Cl}$ at two temperatures, (a) $T=25 \mathrm{~K}$ and (b) $13 \mathrm{~K}$. The full lines are fits by the Davidson-Cole expression (equation (1)). The error bars are obtained from the standard deviation of admittance and scale with inverse frequency.

The complex dielectric spectra of $\kappa-\mathrm{Cl}$ were measured within the molecular planes, $\mathbf{E} \| a c$, at temperatures of $10-50 \mathrm{~K}$ by employing an $L C R$ meter in the range $20 \mathrm{~Hz}-1 \mathrm{MHz}$. The measurement results in the spectrum of complex admittance $Y(\omega)$ proportional to the complex conductivity, $\sigma(\omega)=(l / S) Y(\omega)$, where $l$ is the sample length and $S$ the cross section. Improving upon previous investigations [6], we determined the stray-impedance background contributions of the sample holder $Y_{\mathrm{bg}}(\omega)$ in the open circuit configuration and subtracted them from the sample measurements, which allows us to resolve dielectric relaxation modes near the boundary of the experimental frequency window. Complex dielectric spectra $\varepsilon=\varepsilon^{\prime}-\mathrm{i} \varepsilon^{\prime \prime}$ were extracted from the measured admittance $Y_{\exp }$ using the relation $\varepsilon(\omega)=1+(l / S)\left[Y_{\exp }(\omega)-Y_{\mathrm{bg}}(\omega)-G_{0}\right] /\left(\mathrm{i} \omega \varepsilon_{0}\right)$, where $G_{0}$ is the frequency-independent real conductance of free carriers and the additive 1 is the contribution of the vacuum. The resulting in-plane dielectric responses $\varepsilon_{\|}(\omega)$ are asymmetric in frequency and deviate strongly from the ideal Debye response (figure 2). In contrast to other charge-ordered systems (see figure 10 in [20]), neither a single Cole-Cole form nor a sum of two offer a satisfactory fit. The Davidson-Cole model

$$
\varepsilon(\omega)-\varepsilon_{\mathrm{HF}}=\frac{\Delta \varepsilon}{\left(1+\mathrm{i} \omega \tau_{0}\right)^{1-\beta}},
$$

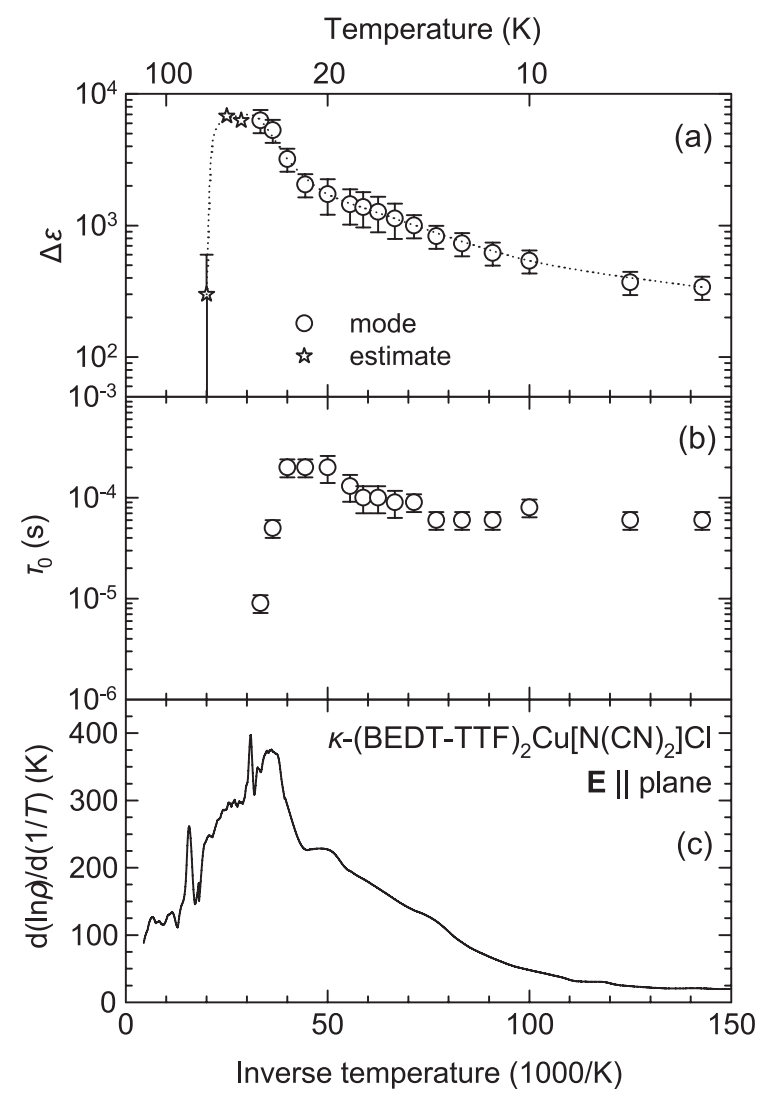

Figure 3. (a) In-plane dielectric strength and (b) mean relaxation time of $\kappa$-(BEDT-TTF $)_{2} \mathrm{Cu}\left[\mathrm{N}(\mathrm{CN})_{2}\right] \mathrm{Cl}$ as a function of inverse temperature. Above $30 \mathrm{~K}$ the mode is outside the experimental frequency window and only the dielectric strength $\Delta \varepsilon_{\|}$can be extracted, denoted by stars. The dotted line is a guide for the eye. (c) The in-plane resistivity derivative shows a broad maximum, which indicates the formation of a low-temperature phase at short-range scale. Dielectric response emerges in the region of resistivity crossover.

commonly used to characterize asymmetric spectra [21], describes our data best. Here $\Delta \varepsilon=\varepsilon_{0}-\varepsilon_{\mathrm{HF}}\left(\varepsilon_{0}\right.$ and $\varepsilon_{\mathrm{HF}}$ are the static and high-frequency dielectric constants, with the latter being negligible), $\tau_{0}$ is the mean relaxation time and $1-\beta$ describes the asymmetric broadening of the relaxation time distribution. Above $30 \mathrm{~K}$ we determine the dielectric strength from the capacitance measured between $10^{5}$ and $10^{6} \mathrm{~Hz}$ (well below the dielectric relaxation), which enables us to track the dielectric mode up to $50 \mathrm{~K}$.

As the temperature drops below $50 \mathrm{~K}$ a very strong relaxational response emerges, measured by the difference between the static and high-frequency dielectric constants $\Delta \varepsilon_{\|} \approx 5000$. From the complex fits of $\varepsilon_{\|}(\omega)$ at a certain temperature we can extract the dielectric strength and mean relaxation time, shown in figures $3(\mathrm{a})$ and (b) as a function of $1 / T$. At very low temperatures the dielectric strength saturates at finite values of about $\Delta \varepsilon_{\|} \approx 300$. The broadening parameter $1-\beta$ of the mode is $0.20 \pm 0.05$. The dielectric mode in $\kappa-\mathrm{Cl}$ does not move with temperature, that is, at least once the dielectric relaxation is well within our experimental frequency window (below $25 \mathrm{~K}$ ). This result excludes the possibility of explaining the observed dielectric relaxation by 
the Maxwell-Wagner effect, which yields an Arrhenius-like behavior of the loss peak. By following our strict experimental procedure [20] we can also rule out any extrinsic effects such as contact resistances and surface layer capacitances.

Next, we argue that the observed behavior cannot be attributed to the hopping of charges which is known to arise in disordered systems. In such a case, the dielectric response is characterized by the absence of a loss peak in the imaginary part and power law in the real part, which decreases with frequency. In contrast, a relaxation-type dielectric response has a low-frequency constant plateau in $\varepsilon^{\prime}$ followed by a decreasing behavior with frequency. Associated with this drop is a Kramers-Kronig-consistent increase in $\varepsilon^{\prime \prime}$, which gives it a characteristic bell-like peak. Both features are obvious for our data shown in figure 2 . In addition, one would expect that the dc conductivity of a system with Anderson localization follows the variable-range hopping law. This is observed neither in our dc conductivity data nor in the literature [10]. Additionally, a power law in frequency would be expected in the ac limit of conductivity. Indeed, a simple indication of the presence of a hopping mechanism would be that the ac conductivity ( $\mathrm{GHz}$ to optical) is significantly enhanced in comparison with the dc conductivity. However, dc and optical conductivities are comparable for temperatures close to the opening of a charge gap. At even lower temperatures the optical conductivity is effectively zero within the charge gap except for the contribution of vibrational features. The in-plane resistivity of $\kappa-\mathrm{Cl}$ at low $T$ is between $10^{6}$ and $10^{8} \Omega \mathrm{cm}$ (figure 1(c)). Seeing that the frequency-dependent power law behavior below $1 \mathrm{MHz}$ is observed only in systems with dc resistivities much larger than $10^{10} \Omega \mathrm{cm}$ [22], the possibility of hopping conductivity can be ruled out.

Quantum electric dipoles were suggested [11] as an explanation of similar observations reported for $\kappa-\mathrm{Cl}$ [10] and other dimerized BEDT-TTF salts $[8,23]$. In order to clarify this point, we have performed comprehensive in- and out-of-plane infrared measurements on $\kappa-\mathrm{Cl}$ single crystals as a function of temperature. The frequencies of certain intramolecular vibrational modes in BEDT-TTF crystals strongly depend on molecular charge, that makes Raman and infrared spectroscopy the superior local probe for the investigation of the charge distribution [24, 25, 18]. Particular emphasis was put on the most charge-sensitive intramolecular vibrational modes $v_{2}\left(\mathrm{a}_{\mathrm{g}}\right), v_{3}\left(\mathrm{a}_{\mathrm{g}}\right)$, and $v_{27}\left(\mathrm{~b}_{1 \mathrm{u}}\right)$. For the two symmetric ones neither a splitting nor any appreciable shift was detected, in full agreement with Raman scattering results [26]. Here we focus on the antisymmetric stretching vibration of the outer $\mathrm{C}=\mathrm{C}$ bond as sketched in figure 4 . Measurements were made by an IR microscope on the thin sides of the crystals in the temperature range down to $12 \mathrm{~K}$ with $1 \mathrm{~cm}^{-1}$ resolution. Due to the strong and linear dependence of the $v_{27}\left(b_{1 u}\right)$ eigenfrequency on the ionicity of the BEDT-TTF molecule of $-140 \mathrm{~cm}^{-1}$ per electron [24, 25], we are able to resolve variations in charge of less than $1 \%$.

In figure 4, the mid-infrared conductivity is plotted for different temperatures. Slightly above $1400 \mathrm{~cm}^{-1}$ we observe the $v_{28}\left(b_{1 u}\right)$ mode, that is split into two (1405 and $1411 \mathrm{~cm}^{-1}$ ) peaks due to Davydov splitting. Since the unit

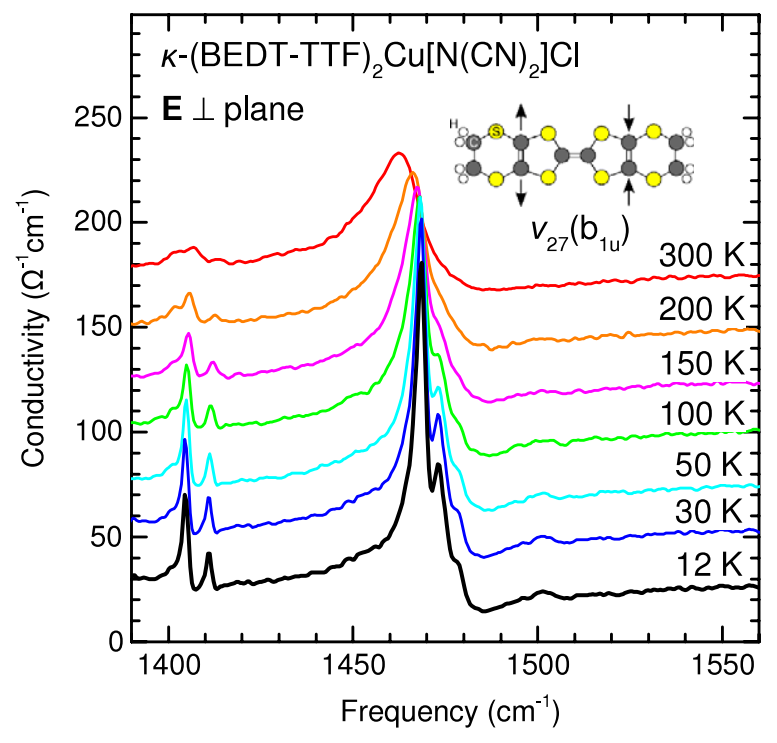

Figure 4. Temperature evolution of the out-of-plane optical conductivity of $\kappa$-(BEDT-TTF $)_{2} \mathrm{Cu}\left[\mathrm{N}(\mathrm{CN})_{2}\right] \mathrm{Cl}$ measured at the narrow side of crystals. For clarity reasons the curves are shifted with respect to each other. Arrows on the sketched BEDT-TTF molecule indicate the antisymmetric $\mathrm{C}=\mathrm{C}$ stretching vibrational mode $v_{27}\left(b_{1 u}\right)$, which is the most sensitive local probe of charge per molecule.

cell contains eight molecules of $\mathrm{D}_{2 h}$ symmetry, the original $\mathrm{b}_{1 \mathrm{u}}$ molecular mode is split into eight branches: $\mathrm{Ag}_{\mathrm{g}}, \mathrm{B}_{1 \mathrm{~g}}, \mathrm{~B}_{2 \mathrm{~g}}$, $\mathrm{B}_{3 \mathrm{~g}}$ (Raman-active only), $\mathrm{A}_{\mathrm{u}}$ (silent), and $\mathrm{B}_{1 \mathrm{u}}, \mathrm{B}_{2 \mathrm{u}}$, and $\mathrm{B}_{3 \mathrm{u}}$ (infrared active along crystal axes) [24]. In our experiment we find two modes of comparable intensity separated by about $6 \mathrm{~cm}^{-1}$ due to the crystallographic inequality of BEDT-TTF molecules in the unit cell. The $v_{27}\left(\mathrm{~b}_{1 \mathrm{u}}\right)$ vibration shows up as a very strong mode at $1460 \mathrm{~cm}^{-1}$, as expected for half a hole per BEDT-TTF molecule [25]. With decreasing temperature there is a slight hardening of a few $\mathrm{cm}^{-1}$ and a strong narrowing, which makes two satellites (separated by 5 and $10 \mathrm{~cm}^{-1}$ ) easier to distinguish at the high-frequency wing of the $v_{27}\left(\mathrm{~b}_{1 \mathrm{u}}\right)$ mode when $T<100 \mathrm{~K}$. As above, the occurrence of these sidebands is explained by the broken equivalence of molecular sites. Note that these features are present at any temperature; there is no qualitative change upon cooling either at $50 \mathrm{~K}$ or at $25 \mathrm{~K}$ when the magnetic ordering takes place. No additional peaks occur above or below the original $1460 \mathrm{~cm}^{-1}$ mode, as expected in the case of charge disproportionation. Basically identical observations are made for the isostructural $\kappa$ - $\mathrm{Br}$ despite large differences in electronic and magnetic properties. We are reassured in our interpretation by the absence of satellites in the spectra of $\kappa$-(BEDT-TTF $)_{2} \mathrm{Cu}_{2}(\mathrm{CN})_{3}$ with a simpler crystal structure [18].

The fact that the $v_{27}\left(\mathrm{~b}_{1 \mathrm{u}}\right)$ molecular vibrational mode does not split when the temperature is lowered leads us to the conclusion that no charge redistribution takes place in $\kappa-\mathrm{Cl}$ exceeding the limit of $0.5 \%$. (Although no static charge disproportionation is observed, oscillations of electronic dipoles faster than $10^{11} \mathrm{~Hz}$ cannot be excluded [18].) For example, a charge disproportionation of $2 \delta \rho=0.6 e$, 
typical for charge-ordered systems such as $\alpha$-(BEDT-TTF $)_{2} \mathrm{I}_{3}$ $[27,20]$, results in a splitting of $80 \mathrm{~cm}^{-1}$. Nothing like this is found, either in our data, or in temperature-dependent Raman and infrared experiments by other groups [26, 24], or in nuclear magnetic resonance data [28].

\section{Discussion}

The task now is to explain how dielectric constants of up to $10^{3}$ can occur although the charge is evenly distributed among all molecules within $\pm 0.005 e$. Can the dielectric relaxation observed in $\kappa-\mathrm{Cl}$ in and out of plane be interpreted as a consequence of low-temperature electronic ferroelectricity established at long-range scales? Can the present theoretical model be modified in order to accomplish the missing charge disproportionation? Are there alternative explanations considering charged domain walls and discommensurations?

The dielectric constants of $\varepsilon_{\|} \approx 10^{4}$ and larger found in one-dimensional electron systems have been successfully attributed to the long-wavelength charge-densitywave excitations [29]. Even values of the order of $\varepsilon_{\|} \approx 10^{9}$ are observed in the spin-density-wave phase of quasi-one-dimensional organic conductors [30]; this is an itinerant antiferromagnetic state with an incommensurate spin-modulation. The electrodynamic properties of this magnetic phase are determined by pinning of the condensate to the background potential created by disorder; this can be understood by invoking the second-order harmonic charge modulation coupled with the antiferromagnetic order of itinerant spins [31, 32]. In the audio- and radio-frequency range the dielectric loss peak is observed with Arrhenius-like resistive decay due to screening by excited charge carriers.

Much less is known about the case of commensurate charge arrangement [33]. The ferroelectric approach was applied to describe charge ordering in the one-dimensional organic compounds (TMTTF) $)_{2} \mathrm{X}$ [34], which at room temperature are dimerized Mott insulators with one hole per dimer on average, similar to $\kappa-\mathrm{Cl}$. The TMTTF molecules are equivalent above the ferroelectric transition, and become inequivalent below due to the alternation of both bonds and site charges. For these systems charge disproportionation was established by NMR and optical spectroscopy [35, 36]. In the latter measurements a $23 \mathrm{~cm}^{-1}$ splitting of the charge-sensitive $v_{28}\left(b_{1 u}\right)$ TMTTF vibrational mode was observed. With a shift of $80 \mathrm{~cm}^{-1}$ per unit charge, this corresponds to a charge disproportionation of $2 \delta \rho=0.29 e$. The charge order transition also becomes apparent in the divergence of dielectric constant right at the transition temperature, reaching values as high as $\Delta \varepsilon=10^{6}$. The two branches of $1 / \Delta \varepsilon_{\|}(T)$ above and below $T_{\mathrm{CO}}$ are very close to linear and the slope below $T_{\mathrm{CO}}$ is approximately twice that above $T_{\mathrm{CO}}$ [37]. From this Curie-Weiss behavior we might extract the Curie constant and estimate the charge disproportionation within a supposed two-molecule dipole. However, the result completely disagrees with the charge disproportionation measured by NMR and optical spectroscopy. This strongly indicates that the dielectric response is not due to the collective behavior of dipole moments associated with TMTTF molecules in the bulk, but caused by solitons localized at domain walls which separate areas of opposite polarization [34]. It is worth recalling the discussion of the neutral-ionic transition in TTF-CA, where the increase of the dielectric constant is associated with domain walls [38]. The domains extend over a few hundred micrometers [39].

In the case of commensurate charge orders in BEDT-TTF salts, the rather small values of the dielectric constant $\varepsilon_{\|}<$ $10^{3}$ are attributed to short-wavelength charge excitations [27, 20, 38], which are usually accompanied by temperatureindependent relaxation times. For the prime example of $\alpha$-(BEDT-TTF $)_{2} \mathrm{I}_{3}$, twinned domains form due to symmetry breaking in the charge-ordered phase $(2 \delta=0.6$ from NMR, Raman and IR measurements [40, 41, 20, 35]), and the motion of domain-wall pairs gives rise to temperature-independent weak dielectric relaxation with $\Delta \varepsilon_{\|} \approx 400$ as well as a negative differential resistance in dc electric fields.

Following this approach, we can estimate charge disproportionation on a BEDT-TTF dimer in $\kappa-\mathrm{Cl}$ from the high-temperature branch of the perpendicular dielectric constant [10], and the extracted value is at least 10 times larger than the upper bound given by our optical measurements. The limited frequency range prevents us from analyzing $\varepsilon_{\|}^{\prime}$ in a similar manner. This consideration again points toward localized domain-wall motion as the excitation responsible for the observed Curie-like peak and the dielectric dispersion, and excludes an ac-field-induced polarization within sample volume.

How can we reconcile the formation of the domain structure in $\kappa-\mathrm{Cl}$ and the dielectric relaxation observed within the dimer Mott phase, on the one hand, with a commensurate magnetic order, on the other hand? The dielectric constant suddenly increases below $50 \mathrm{~K}$, reaches a maximum at $T_{\text {cross }} \approx 40 \mathrm{~K}$ and decreases to lower temperatures. $T_{\text {cross }}$ corresponds well to the broad maximum of the in-plane resistivity derivative, as shown by figure 3(c). The appearance of a dielectric response coincides with the onset of the finite charge gap at $50 \mathrm{~K}$ and the enhancement of the antiferromagnetic spin correlations [42, 19]. Hence, we interpret the broad dielectric feature as a crossover into a new low-temperature phase which develops at short length scales. Nuclear magnetic and electron spin resonance [5, 17, 43] find a weak ferromagnetic state below $T_{\mathrm{N}} \approx 30 \mathrm{~K}$, with substantial short-range fluctuations which extend up to $60 \mathrm{~K}$. Tanatar et al proposed the formation of an antiferromagnetic structure at temperatures as high as $70 \mathrm{~K}$ based on their observation of slow (100 s) resistance relaxation, in both Hall measurements and magnetoresistance [44]. This leads to the picture of magnetic domains which develop below $70 \mathrm{~K}$ and cause the metastable resistance across the domain walls due to soliton formation; the transient effects are largest at about $30 \mathrm{~K}$ and strongly drop below. This result presents a strong indication of an unconventional spin-charge coupling in $\kappa-\mathrm{Cl}$.

Associated with these magnetic domains, we suggest the presence of paired charged defects, which respond to an ac electric field and carry spin as well as charge. At elevated temperatures, fluctuations of commensurate 
antiferromagnetic domains dominate [30]; as the temperature is lowered, however, ferromagnetic domains develop. We attribute the dielectric relaxation to discommensurations that occur in the otherwise commensurate antiferromagnetic phase. Cooling below $50 \mathrm{~K}$, they develop at short length scales; around $20-30 \mathrm{~K}$ the antiferromagnetic phase forms with canted spins at long scales. The dielectric response is then taken over by charged solitons localized within walls separating domains of this ferromagnetic phase. We suggest that the charges accumulate at the domain boundaries in order to promote the formation of magnetic order at long-range scales. Charged topological defects commonly occur in doped correlated insulators and accumulate at the walls separating two domains [34, 45].

The areas of strongly fluctuating electronic dipoles couple to magnetic domains; in this way the spin configuration is linked to the charge degree of freedom without the presence of static charge disproportionation in the bulk. Any associated macroscopic intra- and/or intermolecular distortions and related charge disproportionations are expected to be minute and thus cannot be detected by vibrational spectroscopy of the BEDT-TTF molecules [38]. The broad distribution of relaxation times for dielectric relaxation indicates that it happens between different metastable states, which correspond to local changes of the spin configuration. In analogy with spin-density waves, we expect that the domain-wall motion depends on the interaction with pinning centers, which themselves are randomly distributed in the case of incommensurate ordering, as well as commensurate with the order of commensurability larger than three. The amplitude of the dielectric constant is linked to the density of collective excitations. Hence from the temperature behavior we can conclude that the number of domain walls decreases upon cooling. Their dynamics becomes more restricted and eventually constant below $10 \mathrm{~K}$.

A final remark concerns current theoretical approaches worked out for dimerized $\kappa$-BEDT-TTF systems in general. Hotta and Naka et al independently suggested that quantum electric dipoles on the dimers interact with each other through the dipolar-spin coupling $[11,12]$. The experimentally observed enhancement of the dielectric constant and rise of magnetic correlations below $50 \mathrm{~K}$ indeed indicate the increasing coupling between fluctuating spin and charge degrees of freedom. We propose that short-ranged domains with equivalent configurations of electric dipoles strongly fluctuating faster than $10^{-11} \mathrm{~s}$ [18] may develop concomitantly with antiferromagnetic domains upon cooling; however, the development of charge order and ferroelectricity at long-range scales are suppressed in the weak ferromagnetic phase at low temperatures. This scenario is supported by theoretical consideration by Naka et al [12] showing that the charge susceptibility increases in the paramagnetic phase but suddenly drops when the magnetic phase is formed. Moreover, magnetic and ferroelectric phases are exclusive to each other, also implying that the formation of charge order eliminates the magnetic phase. The latter is in line with results by Hotta [11] revealing that the tendency toward charge order formation strongly diminishes magnetic correlations. The suppression of long-range ferroelectricity may explain why a ferroelectric hysteresis was observed only in the vicinity of the magnetic transition and not at lower temperatures, where the ground state is presumably fully developed [10].

\section{Conclusions}

Optical and NMR spectroscopies rule out any charge disproportionation in the bulk of $\kappa$-(BEDT-TTF $)_{2} \mathrm{Cu}\left[\mathrm{N}(\mathrm{CN})_{2}\right] \mathrm{Cl}$ with antiferromagnetic ground state and canted spins. No appreciable change in the charge-sensitive vibrational features is observed, providing evidence that the charge distribution on BEDT-TTF molecules within dimers is not altered with decreasing temperature. The absence of static electric dipoles on the dimers rules out that ferroelectricity develops at long-range scales at low temperatures as proposed by current models. Rather, the in-plane dielectric response of $\kappa-\mathrm{Cl}$ is explained by short-range discommensurations of the commensurate antiferromagnetic phase in the temperature range $30 \mathrm{~K}<T<50 \mathrm{~K}$, and charged domain-wall relaxations in the weak ferromagnetic state at lower temperatures. Experiments such as (spin-polarized) scanning electron microscopy and transmission x-ray microscopy are a desirable next step due to their ability to visualize charge inhomogeneity, i.e. domains and charge boundaries, and track their formation with temperature. The prominent dielectric relaxation observed in the absence of charge order requires an underlying coupling of charge and spin in the presence of disorder. A microscopic understanding of this coupling calls for further theoretical efforts.

\section{Acknowledgments}

We thank C Hotta, K Kanoda, S Mazumdar, J-P. Pouget, E Rose, M Tanatar, E Tutiš and T Yamamoto for helpful discussions. The project was supported by the Deutsche Forschungsgemeinschaft (DFG), the Deutscher Akademische Auslandsdienst (DAAD) and the Croatian Ministry of Science, Education and Sports under grant 035-00000002836.

\section{References}

[1] Kitaev A 2006 Ann. Phys. 3212

[2] Kanoda K and Kato R 2011 Annu. Rev. Condens. Matter Phys. 2167

[3] Powell B J and McKenzie R H 2011 Rep. Prog. Phys. 74056501

[4] Welp U, Fleshler S, Kwok W K, Crabtree G W, Carlson K D, Wang H H, Geiser U, Williams J M and Hitsman V M 1992 Phys. Rev. Lett. 69840

[5] Kawamoto A, Miyagawa K, Nakazawa Y and Kanoda K 1995 Phys. Rev. B 5215522

[6] Pinterić M, Miljak M, Biškup N, Milat O, Aviani I, Tomić S, Schweitzer D, Strunz W and Heinen I 1999 Eur. Phys. J. B 11217

[7] Smith D F, De Soto S M and Slichter C P 2003 Phys. Rev. B 68024512

[8] Abdel-Jawad M, Terasaki I, Sasaki T, Yoneyama N, Kobayashi N, Uesu Y and Hotta C 2010 Phys. Rev. B 82125119 
[9] Poirier M, Parent S, Côté A, Miyagawa K, Kanoda K and Shimizu Y 2012 Phys. Rev. B 85134444

[10] Lunkenheimer P et al 2012 Nature Mater. 11755 and supplementary information

[11] Hotta C 2003 J. Phys. Soc. Japan 72840

Hotta C 2010 Phys. Rev. B 82241104

Hotta C 2012 Crystals 21155

[12] Naka M and Ishihara S 2010 J. Phys. Soc. Japan 79063707

[13] Tocchio L F, Parola A, Gros C and Becca F 2009 Phys. Rev. B 80064419

[14] Gomi H, Imai T, Takahashi A and Aihara M 2010 Phys. Rev. B 82035101

[15] Li H, Clay R T and Mazumdar S 2011 J. Phys.: Condens. Matter 22272201

Dayal S, Clay R T, Li H and Mazumdar S 2011 Phys. Rev. B 83245106

[16] Shinaoka H, Misawa T, Nakamura K and Imada M 2012 J. Phys. Soc. Japan $\mathbf{8 1} 034701$

[17] Yasin S, Dumm M, Salameh B, Batail P, Meźière C and Dressel M 2011 Eur. Phys. J. B 79383

[18] Sedlmeier K, Elsässer S, Beyer R, Wu D, Ivek T, Tomić S, Schlueter J A and Dressel M 2012 Phys. Rev. B 86245103 and references therein

[19] Elsässer S, Wu D, Dressel M and Schlueter J A 2012 Phys. Rev. B 86155150

[20] Ivek T, Korin-Hamzić B, Milat O, Tomić S, Clauss C, Drichko N, Schweitzer D and Dressel M 2011 Phys. Rev. B 83165128 and references therein

[21] Jonscher A K 1983 Dielectric Relaxation in Solids (London: Chelsea Dielectrics)

[22] Dyre J C and Schrøder T B 2000 Rev. Mod. Phys. 72873

[23] Iguchi S, Sasaki S, Yoneyama N, Taniguchi H, Nishizaki T and Sasaki T 2013 Phys. Rev. B 87075107

[24] Maksimuk M, Yakushi K, Taniguchi H, Kanoda K and Kawamoto A 2001 J. Phys. Soc. Japan 703728

[25] Yamamoto T, Uruichi M, Yamamoto K, Yakushi K, Kawamoto A and Taniguchi H 2005 J. Phys. Chem. B 10915226

Girlando A 2011 J. Phys. Chem. C 11519371

[26] Truong K D, Jandl S, Danilovic B and Poirier M 1999 Synth. Met. 104157

Eldridge J E, Lin Y, Wang H H, Williams J M and Kini A M 1998 Phys. Rev. B 57597
Truong K D, Jandl S and Poirier M 2007 Synth. Met. 157252

Lin Y, Eldridge J E, Schlueter J, Wang H H and Kini A M 2001 Phys. Rev. B 64024506

[27] Ivek T, Korin-Hamzić B, Milat O, Tomić S, Clauss C, Drichko N, Schweitzer D and Dressel M 2010 Phys. Rev. Lett. 104206406

[28] Kanoda K 2012 private communication

[29] Grüner G 1988 Rev. Mod. Phys. 601129

[30] Zornoza P, Petkhov K, Dressel M, Biškup N, Vuletić T and Tomić S 2005 Eur. Phys. J. B 46223

[31] Tomić S, Cooper J R, Jerome D and Bechgaard K 1989 Phys. Rev. Lett. 62462

[32] Maki K and Virosztek A 1989 Phys. Rev. B 399640

[33] Dressel M, Drichko N and Kaiser S 2010 Physica C 470 S589

[34] Brazovskii S A 2008 Ferroelectricity and charge ordering in quasi-1D organic conductors The Physics of Organic Superconductors and Conductors ed A Lebed (Berlin: Springer) pp 313-55

[35] Takahashi T, Nogami Y and Yakushi K 2006 J. Phys. Soc. Japan 75051008 and references therein

[36] Dressel M, Dumm M, Knoblauch T and Masino M 2012 Crystals 2528 and references therein

[37] Monceau P, Nad F Y and Brazovskii S 2001 Phys. Rev. Lett. 864080

[38] Tokura Y, Koshihara S, Iwasa Y, Okamoto H, Komatsu T, Koda T, Iwasawa N and Saito G 1989 Phys. Rev. Lett. 632405

[39] Kishida H, Takamatsu H, Fujinuma K and Okamoto H 2009 Phys. Rev. B 80205201

[40] Kakiuchi T, Wakabayashi Y, Sawa H, Takahashi T and Nakamura T 2007 J. Phys. Soc. Japan 76113702

[41] Yamamoto K, Kowalska A A and Yakushi K 2010 Appl. Phys. Lett. 96122901

[42] Kézsmárki I, Shimizu Y, Mihály G, Tokura Y, Kanoda K and Saito G 2006 Phys. Rev. B 74201101

[43] Miyagawa K, Kanoda K and Kawamoto A 2004 Chem. Rev. 1045635

[44] Tanatar M A, Ishiguro T, Ito H, Kubota M and Saito G 1997 Phys. Rev. B 5512529

[45] Teber S, Stojkovic B P, Brazovskii S A and Bishop A R 2001 J. Phys.: Condens. Matter 134015 